\title{
Leider nur ein Leichtgewicht: Der Text+Kritik-Sammelband zu Christoph Ransmayr enttäuscht
}

\author{
Markus Oliver SPITZ \\ Dr.; Chambre de Commerce, Luxemburg; \\ E-Mail: langpro1@outlook.com
}

\begin{abstract}
It has been more than thirty years since Christoph Ransmayr published The Last World to great success despite its intellectually challenging theme, the fictitious search for Publius Ovidius Naso, known as Ovid, who had been sent into exile by emperor Augustus. Ever since then, his writing has attracted the attention of the public, literary critics and scholars alike. Volume 220 of the renowned edition text + kritik has been dedicated entirely to his work. Its editor, Doren Wohlleben, gathered half a dozen contributions meant to map the different intercultural narrative spaces, which are mainly identifiable in the author's novels and prose writings.
\end{abstract}

Keywords: Ransmayr - Austrian fiction - novel - poetics - criticism

Erster Eindruck: Der Band fällt angesichts des Oeuvres und der Bedeutung Ransmayrs mit gerade einmal achtzig Seiten nebst einer Auswahlbibliografie von Henrike Serfas sehr schmal aus. Das Verzeichnis der Beitragenden weist eine an sich gelungene Mischung aus etablierten Germanistinnen und Germanisten einerseits und dem akademischen Nachwuchs andererseits auf. Man fragt sich jedoch, ob der Aufruf an potenzielle Beitragende seitens der Gastherausgeberin und Heisenberg-Stipendiatin Doren Wohlleben breit genug gestreut wurde. Sicherlich hätten 
sich weitere, gerade auch internationale und in der RansmayrForschung ausgewiesene Mitarbeiter gewinnen lassen.

Man beginnt mit der kritischen Lektüre und stößt auf das erstmals erscheinende Prosagedicht Ballade von der Glücklichen Rückkehr. Dieses Formexperiment bleibt jedoch bis auf einen einzigen Absatz im anschließenden Beitrag Wohllebens zu Ransmayr als „Kalligraph und Kartograph” unkommentiert und somit lose im Raum stehen. (Gleiches gilt für Das Wasserherz, die Rede Ransmayrs bei der Entgegennahme des FontanePreises 2014.) Stattdessen thematisiert Wohlleben auf einigen mageren Seiten die „Flüchtigkeit der Schrift”. Ihre eigenen Forschungsinteressen, beispielsweise Kulturhermeneutik und Antiken-Rezeption, hätten die Auswahl leiten können, fließen so aber bestenfalls in Ansätzen in den Band ein, was bedauerlich ist, denkt man zum Beispiel an die Darstellung der Antike oder Nasos sowie an die Mythen-Rezeption in Die letzte Welt.

Leider gönnt Wohlleben dem Leser auch keine Einleitung, welche die Auswahl und thematische Anordnung der Beiträge hätte begründen können. Stattdessen gibt es eine „Podiumsdiskussion" zusammen mit Jan Bürger und Sigrid Löffler. Letztere ist an dieser Stelle in ihrem Element, wenn sie im Rekurs auf ihre Vertrautheit mit Ransmayr und dessen Werk zum Beispiel auf die „Verräumlichung” des Erzählens verweist. Die „Streifzüge" wirken aber aufs Ganze gesehen nurmehr wie anekdotische Stichwortgeber für das, was eigentlich hätte erfolgen müssen, aber weitgehend unterbleibt, nämlich die tiefgreifende Analyse von Ransmayrs ,kunstvoller Sprache”.

Selbst Bernhard Fetz, mit mehreren Veröffentlichungen zum Thema, beispielsweise zu Strahlender Untergang, hervorgetreten, beschränkt sich auf „Stichwörter” zu einer Poetik. Sein Titel hält allerdings, was er verspricht, indem mehrere werkrelevante Aspekte wie die Darstellung von Geschichte als eines ,gewaltsamen Prozesses der Verdrängung”, die „kritisch-utopische Funktion der Erzählung", die Bedeutung von Wirklichkeit als 
„Imaginationsraum” der Fiktion, „entsubjektivierte Wahrnehmung” und schließlich „Anachronismen” angeschnitten werden. Alexander Honold dekliniert die verschiedenen „Spielarten der Versteinerung" und des Gestaltwandels in Die letzte Welt und Der fliegende Berg, während sich Friedmann Harzer der Zeitgestaltung hauptsächlich in Cox oder Der Lauf der Zeit widmet. Eindeutig essayistisch geprägt ist hingegen Heike Gfrereis' „Poesie in Prosa”, welche kein Wort über die Ballade verliert. Martin Pollacks „Alphabetische Suppe und JubiläumssalutHohenzollern-Torte" mutet weit eher wie eine leidlich unterhaltsame Laudatio denn eine kritische Betrachtung an.

Es ist immer unfair, einen Sammelband mit einem anderen zu vergleichen, aber hier sei dies dennoch gestattet, um den Beleg dafür zu führen, dass diese Veröffentlichung eindeutig nicht die Breite, Tiefe und Gewichtigkeit vergleichbarer Publikationen, beispielsweise Bis zum Ende der Welt, 2015 von Attila Bombitz im Rahmen der „Österreich-Studien” herausgegeben, aufweist. Dies ist auch der Tatsache geschuldet, dass das vonseiten der Herausgeberschaft vorgegebene Grundmotiv des "Gehens" sowie die Thematik des Auskundens ,interkultureller, existenzieller Erzählräume", welche trotz ihrer Abstraktheit immer noch ein roter Faden hätten sein können, hier nach dem Sandwich-Prinzip zwischen Essays geschoben sind, welche allenfalls marginalen Bezug auf die genannten Aspekte nehmen. Somit bleibt das einzige Gehen in einigen Beiträgen das Verlorengehen in Versatzstücken, welche sich nicht zu einem Ganzen fügen wollen.

Doren Wohlleben (Hg.): Christoph Ransmayr (text+kritik 220). München: edition text+kritik 2018, $91 \mathrm{~S}$. 